\title{
Modèle global pluie-débit pour la prévision et la prédétermination des crues
}

\author{
Overall rain-flow model for flood forecasting and pre-determination
}

\author{
par R. Garçon
}

EDF - Ressources en Eau

The water flow mechanisms in a catchment area are highly complex and detailed modelling of these mechanisms is obstructed by the problem of understanding the phenomena as well as that of gaining accurate knowledge of the many local sensitive parameters. Faced with this difficulty, and without wanting to cast doubt on the hope of future progress, one of the hydrologist's solutions to assist decision making in operational conditions, has been to develop simple models, dealing only with the main traits of the rain-flow transformation process. For example, those governing the evolution of the percentage of rain which flows away rapidly or the time dynamics of flood nun-off. Here, with respect to these two aspects, we draw a parallel between the models retained by EDF for three different purposes: short term flood forecasting (DPFT), estimation of the probability of extreme floods (GRADEX) and continuous monitoring of water resources (MORDOR). Particular emphasis is placed on the way in which each of these models takes account of the move from normal to extreme conditions.

\section{I INTRODUCTION}

\subsection{Les crues pour le producteur d'électricité}

Les crues, normales ou extrêmes, ont toujours été un sujet de grand intérêt - ou de préoccupation - pour EDF. Cet excédent d'eau constitue, bien sûr, une ressource qu'il convient de gérer correctement pour sa valeur énergétique. Mais il peut être également synonyme de menace pour l'équipement de production. De plus, une défaillance au niveau des installations hydroélectriques ou de leur conduite risque d'aggraver les effets des crues à leur aval. On doit donc, en premier lieu, prendre en compte le phénomène de crue pour dimensionner les capacités d'évacuation des barrages et mettre au point leurs consignes d'exploitation en crue. Pendant la crue elle-même, on s'attachera à prévoir l'évolution du débit entrant dans la retenue pour la meilleure application possible de la consigne. de crue. Cette consigne fait bien entendu passer la sécurité au premier plan. Toutefois, avant et après la crue, il importera pour le producteur d'électricité de suivre avec la plus grande précision l'état hydrique des bassins versants exploités pour anticiper sur les apports futurs et gérer ses stocks d'énergie en conséquence.

\subsection{Quelle modélisation des crues pour EDF ?}

La bonne prise en compte du phénomène de crue est donc nécessaire dans chacune de ces trois fonctions : prédétermination des crues extrêmes, prévision à court terme des débits et suivi en continu des ressources en eau. Le contexte indus- triel de cette aide à la décision pour laquelle l'hydrologue est sollicité l'oblige à rechercher des méthodes tournées vers la robustesse et la possibilité de mise en œuvre dans des conditions opérationnelles. Le titre de ce colloque est d'ailleurs centré sur une des dimensions de la robustesse attendue : par leur nature fréquente, ce sont les crues " normales " qui fournissent la base principale de l'apprentissage de I'hydrologie de crue. Mais la préoccupation d'EDF ou des autres acteurs ne se limite pas aux crues normales et il appartient à l'hydrologue de bien choisir dans son appréhension du phénomène les caractères extrapolables aux crues extrêmes.

Pour le reste, cette particularité du contexte amènera I'hydrologue à privilégier des solutions pragmatiques. Il ne s'agit pas ici de démonter pièce par pièce le processus de formation des crues en partant de l'échelle de la goutte d'eau. Si les progrès dans la connaissance des mécanismes élémentaires sont de nature à favoriser, à terme, ceux des outils opérationnels d'aide à la décision, il n'en reste pas moins qu'on se heurte pour l'instant à quelques obstacles : la complexité des processus hydrologiques, la difficulté à faire cohabiter dans ce domaine différentes échelles de temps et d'espace dans une même modélisation, l'impossibilité économique de décrire avec exhaustivité les caractéristiques d'un bassin à une échelle fine et, encore plus, de valider en temps réel les résultats d'un modèle à cette même échelle. La tendance actuelle en matière de mesures de terrain est hélas, même en présence d'enjeux industriels, à 
chercher plutôt à faire aussi bien à un moindre coût qu'à faire plus et mieux. Ces contraintes orientent donc l'aide à la décision vers une approche assez globale du processus.

Précisons le type de phénomène dont nous allons parler ici. Les bassins versants à l'amont des prises d'eau et barrages exploités par EDF sont en zone rurale ou naturelle et ont des superficies allant de quelques $\mathrm{km}^{2}$ à quelques dizaines de milliers de $\mathrm{km}^{2}$. Nous viserons plus particulièrement les bassins versants de montagne ou moyenne montagne dont la taille est comprise entre 100 et $10000 \mathrm{~km}^{2}$. En France, de tels bassins présentent la caractéristique assez répandue de voir leurs débits de crue formés pour l'essentiel par le ruissellement, direct ou peu différé, des précipitations liquides. La fonte de la neige ou un débit de base élevé, consécutif à une longue période humide, peuvent contribuer de façon significative au volume des crues, mais cet apport est - relativement - limité, notamment dans le cas des crues les plus fortes. Cette caractéristique est d'autant moins vérifiée que les bassins versants sont vastes, peu pentus, perméables et situés dans des régions froides.

\subsection{Le concept classique production-transfert}

Dans les bassins qui nous intéressent, le processus d'écoulement rapide de l'eau de pluie est donc l'objet principal de l'hydrologie de crue. En restant à une échelle globale, pour les raisons énoncées plus haut, on peut aisément concevoir un processus en deux étapes :

- La précipitation liquide se répartit d'abord en deux fractions. La première rejoint dans un délai assez bref le réseau hydrographique. La seconde est absorbée ou infiltrée, destinée par la suite à s'évaporer ou à contribuer à la composante lentement évolutive du débit, le "débit de base".

- La lame d'eau disponible pour un écoulement rapide est acheminée dans le bassin jusqu'à son exutoire selon un processus gouvernant à la fois le temps de transfert et l'amortissement de l'onde de crue.

Cette conceptualisation production-transfert est à la base des outils classiques de l'hydrologue. On la retrouve dans les modèles génériques utilisés par EDF dans chacune des 3 applications de l'hydrologie évoquées plus haut. Peut-on dégager, pour les deux étapes, des principes communs repris par 3 modèles répondant à 3 questions différentes ? Ces principes résistent-ils bien au passage de la normale à l'extrême ? Quelles sont les limites de la qualité de la modélisation si l'on s'en tient à l'application de ces principes de base et quels axes de progrès se dessinent à court terme pour l'hydrologie opérationnelle?

\subsection{Trois modèles, un même cas d'étude}

Nous allons tenter d'apporter quelques réponses à ces questions en observant le fonctionnement des 3 modèles sur des cas concrets. Pour compenser notre ambition sur le nombre de modèles examinés, nous allons pour le reste nous en tenir à une unité de lieu et de temps. Nous avons sélectionné le bassin du Verdon à l'amont du barrage de Castillon.

Ce barrage contrôle un bassin versant de $655 \mathrm{~km}^{2}$ de forme allongée. C'est un bassin de montagne : plus de $3000 \mathrm{~m}$ d'altitude pour les plus hauts sommets, $880 \mathrm{~m}$ à la retenue de Castillon. Tourné vers la Méditerranée et exposé aux remontées de masses d'air chaudes et humides génératrices de pluies abondantes, ses fortes pentes favorisent un écoulement rapide. Ce bassin est, en outre, important pour EDF compte tenu de la capacité énergétique de la retenue de Castillon. " lac de tête " du Verdon. Il n'est donc pas étonnant que l'on soit en mesure d'examiner ce bassin à la lueur des modélisations correspondant aux 3 besoins fondamentaux d'EDF (prédétermination des crues extrêmes, prévision à court terme, suivi de la ressource en eau).

L'autre raison qui nous a amenés à choisir ce bassin tient à la réalisation d'un événement marquant dans les années récentes. En effet, le 5 novembre 1994, une crue importante mit un point d'orgue à un automne particulièrement pluvieux. Peut-on dire pour autant qu'un tel événement soit un point de repère satisfaisant pour illustrer la problématique du passage à "I'extrême" ? Il faudrait avoir pour cela une définition précise de l'adjectif. On ne peut se référer au sens de limite absolue parfois attribué au mot extrême. On ne saurait, en effet, pour aucun événement hydrologique déjà recensé à l'exception, peut-être, du Déluge - certifier qu'il ne puisse être un jour dépassé. Nous considérerons plutôt ici extrême comme l'opposé de normal, ordinaire. Comme cela ne nous fournit pas un critère très objectif, nous verserons dans l'anthropomorphisme pour considérer comme "normal" un événement qui l'est à l'échelle de la vie humaine et comme "extrême" un événement qui aurait de fortes chances de ne pas être dépassé pendant la même durée. De ce point de vue, la durée de retour des premiers ne dépasserait pas 10 ans tandis que celle des seconds ne descendrait pas au dessous de 50 ans. Entre les deux, les spécialistes pourront peut-être identifier le no man's land des phénomènes "rares". L'avantage d'un tel usage du qualificatif "extrême" est qu'il s'adapte bien aux situations occasionnant des dégâts imprévus à des édifices n'ayant pas été conçus pour résister à des événements jamais observés par une génération. Sur ce plan, on peut se souvenir, par exemple, du sort que la crue de novembre 1994 avait réservé à la gendarmerie de Colmars. Il semblerait donc que l'événement mérite par certains aspects l'appellation "extrême", mais cela devrait être confirmé par l'estimation objective de sa durée de retour. Une telle estimation est justement du ressort de la première application dont nous devons parler.

\section{II — L'ESTIMATION DES PROBABILITÉS DE CRUES EXTRÊMES : LE GRADEX}

La méthode du GRADEX [1] a été utilisée plusieurs centaines de fois depuis 30 ans pour estimer les probabilités de crues extrêmes et étayer rationnellement le dimensionnement d'évacuateurs de crue [2] ou la révision des dispositifs en place. Elle vise plus particulièrement le type de bassin qui nous intéresse ici. Le but de ce chapitre n'est pas d'expliciter de $\mathrm{A}$ à $\mathrm{Z}$ cette méthode, l'approche probabiliste de la prédétermination des crues faisant déjà l'objet d'une communication dans le cadre de ce colloque.

\subsection{Analyse en volume}

Rappelons simplement que, du point de vue de la production d'écoulement à partir des pluies, les auteurs de la méthode - et ses utilisateurs - font l'hypothèse que la rétention moyenne d'un bassin versant pour les crues extrêmes 
tend vers une limite finie. Cela implique que le rendement marginal des précipitations abondantes tend vers $100 \%$. L'état hydrique initial du bassin ne peut jouer que sur la vitesse à laquelle ce rendement se rapproche de $100 \%$. Compte tenu d'une première hypothèse sur la loi de probabilité des pluies extrêmes [3,4], il s'ensuit que, sur un graphe de GUMBEL, les fonctions de répartition des maxima annuels des volumes d'eau précipités et écoulés sont, dans la zone des valeurs extrêmes, 2 droites séparées par la rétention moyenne limite.

Appliquée en 1990 au bassin du Verdon [5], cette méthode produisait les résultats présentés sur la figure 1 . La précipitation spatiale est estimée par la moyenne des relevés de Castillon, Allos et Lambruisse.

On retient de cette figure quelques valeurs. A un niveau de fréquence choisi dans la zone des crues ordinaires $(U=-1$, $T=1 /(1-F)=1,07)$, on peut lire que la pluie spatiale dépassée 93 années sur 100 est égale à $44 \mathrm{~mm}$ en 24 heures. A ce même niveau de fréquence, l'écoulement en $24 \mathrm{~h}$ n'est que de $7 \mathrm{~mm}$, pour un "rendement" de $16 \%$. Cela ne signifie pas bien sûr que toutes les pluies de $44 \mathrm{~mm}$ engendrent un écoulement de $7 \mathrm{~mm}$, indépendamment de l'état initial du bassin. Mais cela fixe un point de référence pour le rendement des épisodes "normaux". Et on peut comparer ces valeurs théoriques à celles observées pour un des épisodes mineurs de l'automne 94 . Du 19 au 20 octobre, $27 \mathrm{~mm}$ d'eau sont tombés en 24 heures sur un bassin déjà bien humide mais pas encore saturé et le débit moyen a atteint $30 \mathrm{~m}^{3} / \mathrm{s}$, soit un écoulement de $4 \mathrm{~mm}$ et un rendement de $15 \%$.

Revenons à la figure 1 et déplaçons-nous sur la droite jusqu'aux valeurs décennales : $81 \mathrm{~mm}$ de pluie pour $22 \mathrm{~mm}$ d'écoulement et un rendement passant à $27 \%$. Au-delà de cette valeur, les fonctions de répartition sont extrapolées suivant le gradex des précipitations spatiales en $24 \mathrm{~h}$ de la saison à plus fort risque, l'automne, soit $17,6 \mathrm{~mm}$. Cela nous conduit à une pluie centennale de $122 \mathrm{~mm}$ et à un écoulement centennal de $63 \mathrm{~mm}$ pour un rendement de $52 \%$. Rappelons que le même écoulement centennal pourrait tout aussi bien être produit par une précipitation décennale tombant sur un sol très humide ou, inversement, par une pluie millennale arrivant sur un bassin très sec.

En novembre 1994, la précipitation spatiale en 24 heures centrées sur l'événement a été estimée à 102,6 mm d'après les mesures faites aux 3 postes cités plus haut. Cette valeur correspond, statistiquement, à un équivalent "non centré" de $90 \mathrm{~mm}$, après application du coefficient de WEISS, soit à un événement presque vingtennal. D'après les bilans réalisés au barrage de Castillon, l'écoulement en $24 \mathrm{~h}$ aurait atteint $54 \mathrm{~mm}$, soit $60 \%$ de la pluie, valeur de rendement élevée qui témoigne de l'état initial très humide du bassin suite aux pluies abondantes de septembre et octobre. Cela expliquerait qu'une pluie de durée de retour 20 ans ait engendré un écoulement de durée de retour voisine de 55 ans.

\subsection{Analyse en débit de pointe}

La sévérité d'une crue est assez rarement quantifiée par son seul volume. Le plus souvent, c'est son débit de pointe qui fait référence et la méthode du GRADEX permet d'attribuer une durée de retour à une crue d'après la valeur du débit maximum instantané enregistré pendant l'événement. Elle fait pour cela l'hypothèse que le rapport entre débit de pointe et débit moyen, bien que variable d'une crue à l'autre, est, en moyenne, indépendant du volume de celles-ci. Elle propose alors de passer du débit moyen d'une durée de retour donnée au débit de pointe de même durée de retour par application du coefficient de forme moyen déduit d'un échantillon de crues "normales" (celles à disposition de l'hydrologue). Dans l'étude de 1990, le coefficient de forme retenu au vu des hydrogrammes de crues disponibles a été 1,70 , soit un peu plus que la moyenne empirique des coefficients calculés (fig. 2).

La crue du 5 novembre 1994 a eu, à Castillon, une pointe estimée à $620 \mathrm{~m}^{3} / \mathrm{s}$ d'après les données collectées au barrage pendant la crue. Son coefficient de forme $(1,53)$ légèrement inférieur à la moyenne s'explique par la durée de l'épisode pluvieux générateur.

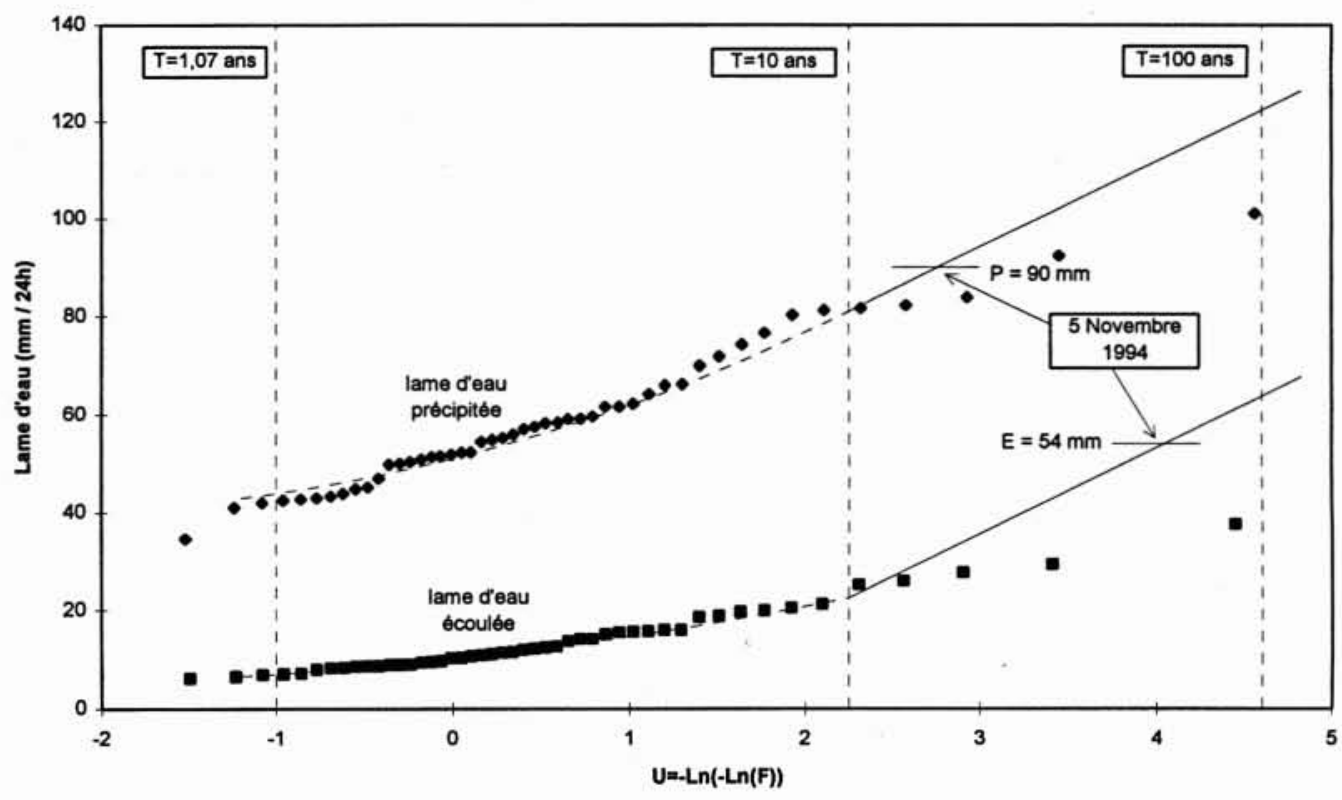

1. Le Verdon à

Castillon : maxima annuels de la lame d'eau journalière. 


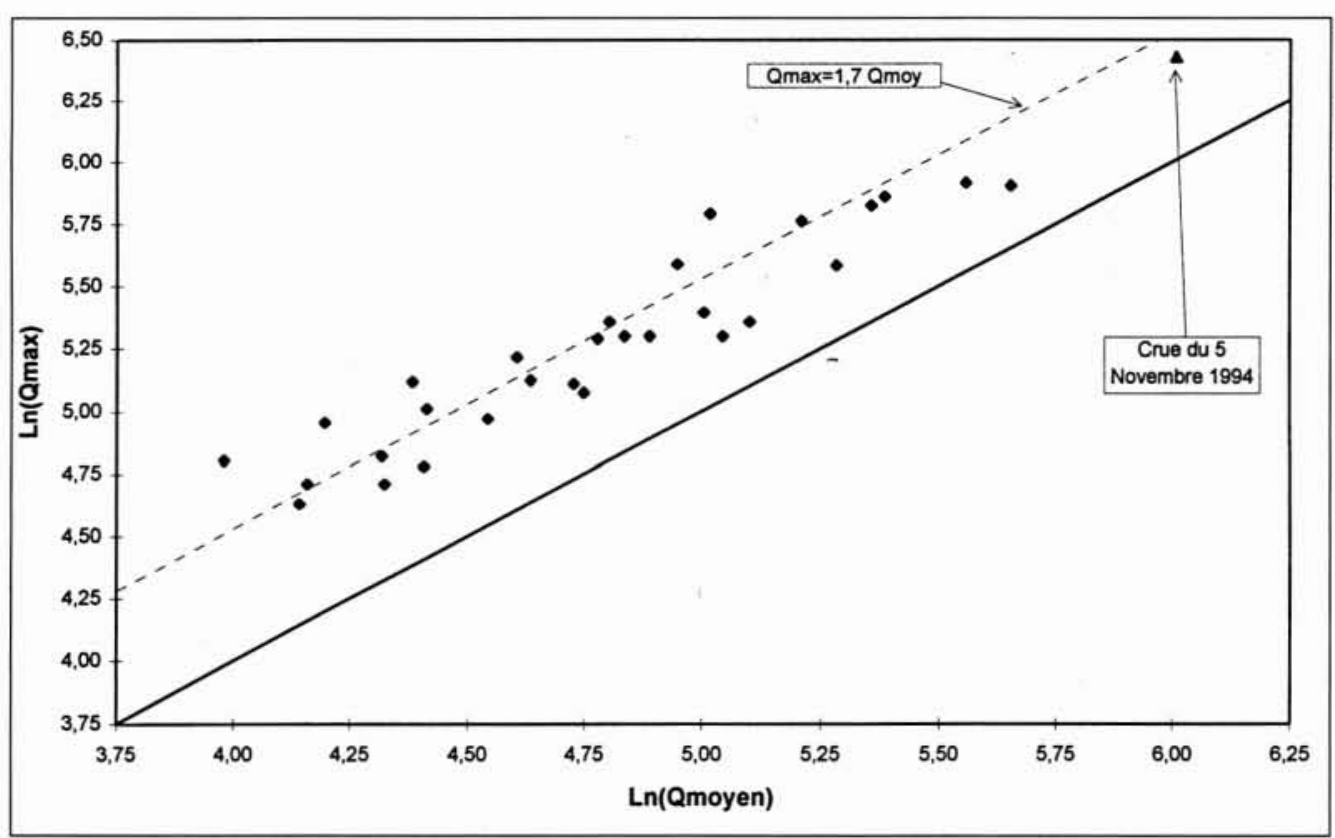

2. Comparaison des débits moyens journaliers et maximaux instantanés à Castillon.

\begin{tabular}{|c|c|c|c|c|c|c|c|c|c|}
\hline Evénement & $\mathrm{Pj}$ & $\mathrm{Tp}$ & $\mathrm{Ej}$ & $\mathrm{Te}$ & $\mathrm{Qj}$ & "Rétention" & "Rendement" & Qmax & Qmax/Qj \\
\hline $20 / 10 / 94$ & $27 \mathrm{~mm}$ & faible & $4 \mathrm{~mm}$ & faible & $30 \mathrm{m3} / \mathrm{s}$ & $23 \mathrm{~mm}$ & $15 \%$ & $\sim 40 \mathrm{~m} 3 / \mathrm{s}$ & $\sim 1,33$ \\
\hline $\mathrm{F}=0,065$ & $44 \mathrm{~mm}$ & 1,07 an & $7 \mathrm{~mm}$ & 1,07 an & $52 \mathrm{~m} 3 / \mathrm{s}$ & $37 \mathrm{~mm}$ & $16 \%$ & $88 \mathrm{~m} 3 / \mathrm{s}$ & 1,7 \\
\hline $\mathrm{F}=0,9$ & $81 \mathrm{~mm}$ & 10 ans & $22 \mathrm{~mm}$ & 10 ans & $170 \mathrm{m3} / \mathrm{s}$ & $59 \mathrm{~mm}$ & $27 \%$ & $290 \mathrm{~m} 3 / \mathrm{s}$ & 1,7 \\
\hline $05 / 11 / 94$ & $90 \mathrm{~mm}$ & 17 ans & $54 \mathrm{~mm}$ & 55 ans & $406 \mathrm{m3} / \mathrm{s}$ & $36 \mathrm{~mm}$ & $60 \%$ & $620 \mathrm{~m} 3 / \mathrm{s}$ & 1,53 \\
\hline $\mathrm{F}=0,99$ & $122 \mathrm{~mm}$ & 100 ans & $63 \mathrm{~mm}$ & 100 ans & $480 \mathrm{m3} / \mathrm{s}$ & $59 \mathrm{~mm}$ & $52 \%$ & $820 \mathrm{~m} 3 / \mathrm{s}$ & 1,7 \\
\hline
\end{tabular}

Tableau I. - Caractéristiques des différents événements réels ou virtuels.

Le tableau 1 récapitule les caractéristiques des différents événements, réels ou virtuels, évoqués plus haut.

Finalement, avec ses hypothèses simples, la méthode du GRADEX fournit un guide de lecture satisfaisant pour les niveaux de crue atteints. La durée de retour estimée pour la crue de 1994 - 40 ans en pointe et 55 ans en volume - n'est pas en contradiction avec l'analyse des crues historiques qui révèle que 2 crues en 1843 et 1886 ont été comparables ou supérieures à la crue de novembre 1994.

\section{III — LA PRÉVISION DES CRUES À COURT TERME : LA DPFT}

La prévision des débits de crue par une approche pluiedébit est la solution qui permet le plus d'anticipation, la prévision par propagation sacrifiant à la précision le délai de concentration des bassins en amont des premières stations hydrométriques. La méthode DPFT $[6,7]$ est une approche pluie-débit reposant explicitement sur le concept productiontransfert. Reprenant le principe de l'"hydrogramme unitaire", elle postule l'existence d'une fonction de transfert temporelle moyenne, linéaire, caractéristique du bassin, transformant une intensité de pluie spatiale nette - ou efficace - en ruissellement à l'exutoire. Un algorithme astucieux permet d'identifier cette fonction de transfert directement sans postulat $a$ priori sur le processus de production (transformation de la précipitation brute en pluie efficace). La fonction de pro- duction est identifiée en aval. EDF surveille en temps réel, au moyen de ce modèle, les crues de 30 bassins différents.

La fonction de production utilisée pour ces applications est une fonction analytique faisant dépendre la pluie efficace pour le ruissellement rapide $P_{E}$ de la pluie brute $P_{B}$ selon la relation : $P_{E}=P_{B}{ }^{2} /\left(P_{B}+D\right), D$ étant un déficit d'écoulement extrême estimé en fonction des conditions hydrologiques antérieures. Ce dernier modèle confère une efficacité nulle aux premières gouttes de pluie, l'efficacité marginale croissant jusqu'à se rapprocher inexorablement des $100 \%$ pour les averses les plus violentes, pour lesquelles l'écart entre $P_{E}$ et $P_{B}$ tend vers $D$.

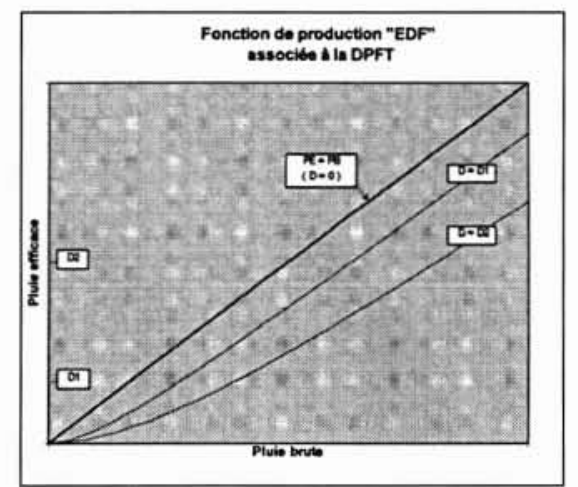

3. Fonction de production "EDF" associée à la DPFT. 


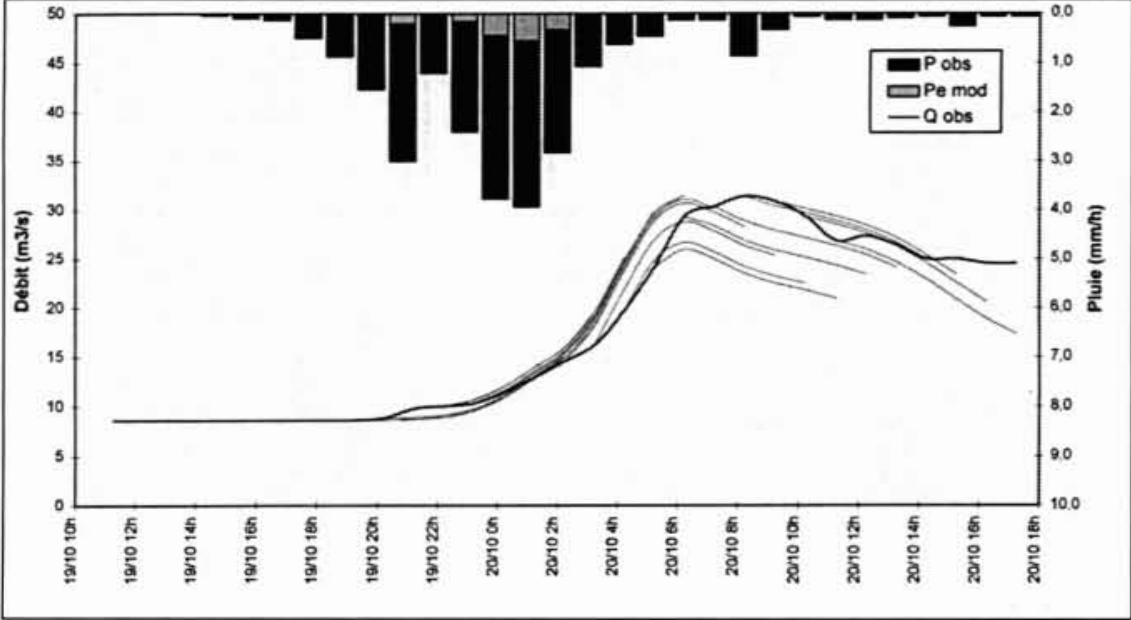

4. Crue du 20 octobre 1994 au Pont de Méouilles $\left(569 \mathrm{~km}^{2}\right)$. Simulations de prévisions à pluie future connue. augmentation importante de l'écoulement différé en cours d'épisode, augmentation qui prend d'ailleurs en défaut le modèle à la décrue.

Pour l'épisode du 5 novembre, l'efficacité modélisée est, en comparaison, très impressionnante : $62 \%$ en moyenne sur 48 heures et plus de $80 \%$ au paroxysme de l'épisode dans la matinée du 5. D'après la figure 5 , le modèle surestime légèrement le rendement. La fonction de transfert paraît, quant à elle, bien adaptée. S'il semble, à la pointe, $\mathrm{y}$ avoir un décalage d'une heure, les perturbations subies par la section de mesure et le détarage qui en découle à ce moment crucial ne permettent pas de trancher. Sur la petite remontée vers $22 \mathrm{~h}$, le

Un tel modèle était en service en 1994 sur le Haut Verdon [8] et il est possible de simuler aujourd'hui son fonctionnement pour les 2 crues qui nous ont déjà servi de points de repère pour le gradex, celles du 20 octobre et du 5 novembre. Le point de contrôle du débit est, dans ce cas, la station hydrométrique du Pont de Méouilles $\left(569 \mathrm{~km}^{2}\right)$ à l'amont immédiat de la retenue de Castillon. En effet, le bilan, établi au barrage en temps réel, du débit entrant dans une retenue, est souvent imprécis à pas de temps fin - le modèle fonctionne au pas de temps horaire - et une station en rivière est préférable dans ce cas. La pluie spatiale est estimée par moyenne des pluies mesurées à Allos, Thorame et Lambruisse.

Les simulations des 2 épisodes sont reproduites sur les figures 4 et 5 . Il importe de signaler que ce sont des simulations de prévisions à pluie future connue. En temps réel, la qualité des prévisions se dégrade au-delà d'une échéance correspondant au temps de concentration du bassin, compte tenu de notre incapacité à anticiper avec précision l'évolution des précipitations. Cette simulation à pluie future connue est utilisée ici pour mieux apprécier ou critiquer la modélisation du processus pluie-débit.

Pour la crue du 20 octobre, la faiblesse de l'écoulement rapide engendré par les pluies est frappante : $15 \%$ à la pointe et $8 \%$ sur l'ensemble de la journée d'après le modèle qui rend pourtant bien compte du volume observé au Pont de Méouilles, le débit complémentaire étant produit par un écoulement différé de $9 \mathrm{~m}^{3} / \mathrm{s}$ au début de la crue et de $20 \mathrm{~m}^{3} / \mathrm{s}$ à la fin de la crue. Au niveau de l'acheminement de la crue jusqu'à l'exutoire, le modèle prend un peu d'avance sur les réalisations. On peut se demander si cet écart vient d'une propagation plus lente des crues de faible importance ou si la comparaison est faussée par une synchronisme entre modélisation et observation est parfait. La fin de la décrue est mal suivie par le modèle. Ce dernier travaillant, comme son nom l'indique, sur les variations de débit, la surestimation du rendement des pluies entraîne aussi une surestimation des variations de débit négatives. En outre, l'évolution de la composante différée de l'écoulement perturbe le modèle qui a tendance à revenir au débit de base initial. Un traitement de cette composante lentement évolutive du débit a été, depuis, incluse dans le modèle.

Sur ces deux épisodes, la DPFT montre qu'elle arrive à saisir l'essentiel du processus pluie-débit dans une gamme très étendue de phénomènes, les débits de pointe variant dans un rapport de 1 à 15 et le taux d'efficacité des pluies pour l'écoulement rapide couvrant lui-même la quasi-totalité de la plage physique des valeurs de rendements.

\section{IV — LE SUIVI DES RESSOURCES EN EAU : MORDOR}

Depuis quelques années, le Service Ressources en Eau suit l'évolution de l'état hydrique des bassins à fort enjeu énergétique en s'appuyant sur le modèle MORDOR [9]. MORDOR

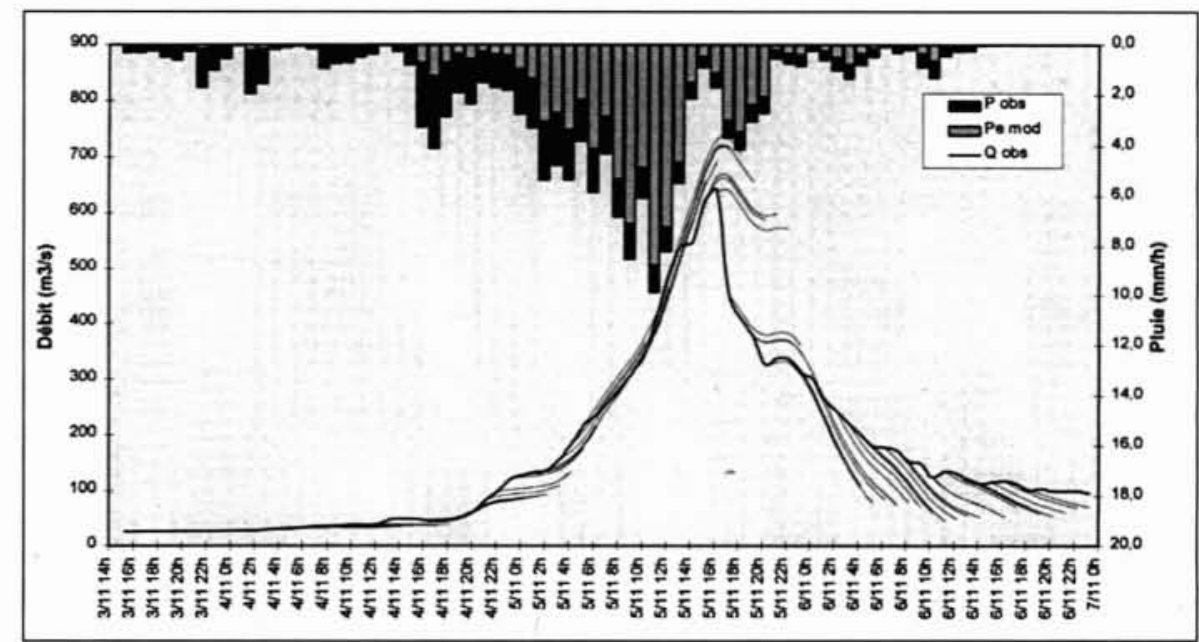

5. Crue du 5 novembre 1994 au Pont de Méouilles $\left(569 \mathrm{~km}^{2}\right)$. Simulations de prévisions à pluie future connue. 


\begin{tabular}{|c|c|c|c|c|c|}
\hline $\begin{array}{c}\text { Séquence } \\
\text { perturbée }\end{array}$ & $\begin{array}{c}\text { Pluie } \\
\text { spatiale }\end{array}$ & $\begin{array}{c}\text { Ecoulement } \\
\text { observé }\end{array}$ & $\begin{array}{c}\text { Rendement } \\
\text { observé }\end{array}$ & $\begin{array}{c}\text { Ecoulement } \\
\text { simulé }\end{array}$ & $\begin{array}{c}\text { Rendement } \\
\text { simulé }\end{array}$ \\
\hline 1 au 3 Septembre & $52 \mathrm{~mm}$ & $2,8 \mathrm{~mm}$ & $5,50 \%$ & $3,9 \mathrm{~mm}$ & $7,50 \%$ \\
\hline 20 au 21 Octobre & $52 \mathrm{~mm}$ & $12,2 \mathrm{~mm}$ & $23 \%$ & $10,2 \mathrm{~mm}$ & $20 \%$ \\
\hline 3 au 15 Novembre & $215 \mathrm{~mm}$ & $186 \mathrm{~mm}$ & $86 \%$ & $164 \mathrm{~mm}$ & $76 \%$ \\
\hline
\end{tabular}

Tableau 2. - Comparaison des écoulements et rendements observés et simulés.

est un modèle à réservoirs simulant schématiquement le parcours de l'eau dans un bassin en s'appuyant sur la donnée quotidienne de la précipitation spatiale et de la température de l'air mesurée en un point de référence. Quoique ses données d'entrée restent rudimentaires, MORDOR met en jeu une modélisation plus complexe que la DPFT ou le GRADEX puisqu'il traite des échanges d'eau entre 5 réservoirs conceptuels dont les niveaux de remplissage constituent les variables d'état du système. Malgré cette relative complexité, il ne s'agit pas d'un modèle physique. Ses paramètres sont calés statistiquement sur les seules mesures de débit à l'exutoire.

Un tel modèle permet la prévision des apports journaliers pour les jours futurs pour autant qu'on dispose de prévisions fiables de la précipitation et de la température de l'air. Il permet également l'élaboration de scénarios d'apports à plus long terme (pour les quelques mois durant lesquels les conditions initiales du bassin ont encore un poids sensible) en partant de scénarios météorologiques issus, par exemple, de chroniques historiques. On peut enfin, sur la base de cette modélisation, situer statistiquement l'état des réserves hydriques du bassin par rapport à la période historique déjà simulée.
La figure 6 montre la simulation par MORDOR de l'automne 1994 à Castillon. Fonctionnant au pas de temps journalier, le modèle ne peut simuler finement la dynamique des crues. Si les pluies du jour sont tombées plutôt en début ou en fin de journée, sa réponse peut sembler "décalée" d'une journée. La propagation de l'écoulement jusqu'à l'exutoire est réalisée par une fonction de transfert linéaire et invariante dont seuls les coefficients à $\mathrm{J}$ et $\mathrm{J}+1$ sont significatifs pour un tel bassin.

L'objectif du modèle, dont on attend une aide à la gestion énergétique des réserves, est plutôt la bonne appréhension des volumes écoulés et stockés par le bassin naturel à l'échelle de périodes perturbées de 1 à 15 jours. De ce point de vue, le tableau 2 montre une réussite partielle du modèle dans une gamme étendue de phénomènes.

Le modèle semble avoir manqué d'eau pour reproduire les débits exceptionnels observés début novembre. Comme il n'a pas stocké d'eau en excès, au vu de la simulation du débit en novembre et décembre, l'interprétation de cette sous-estimation est délicate.

Il est intéressant, au-delà de cette première analyse qui montre la progression quantitative globale du rendement des
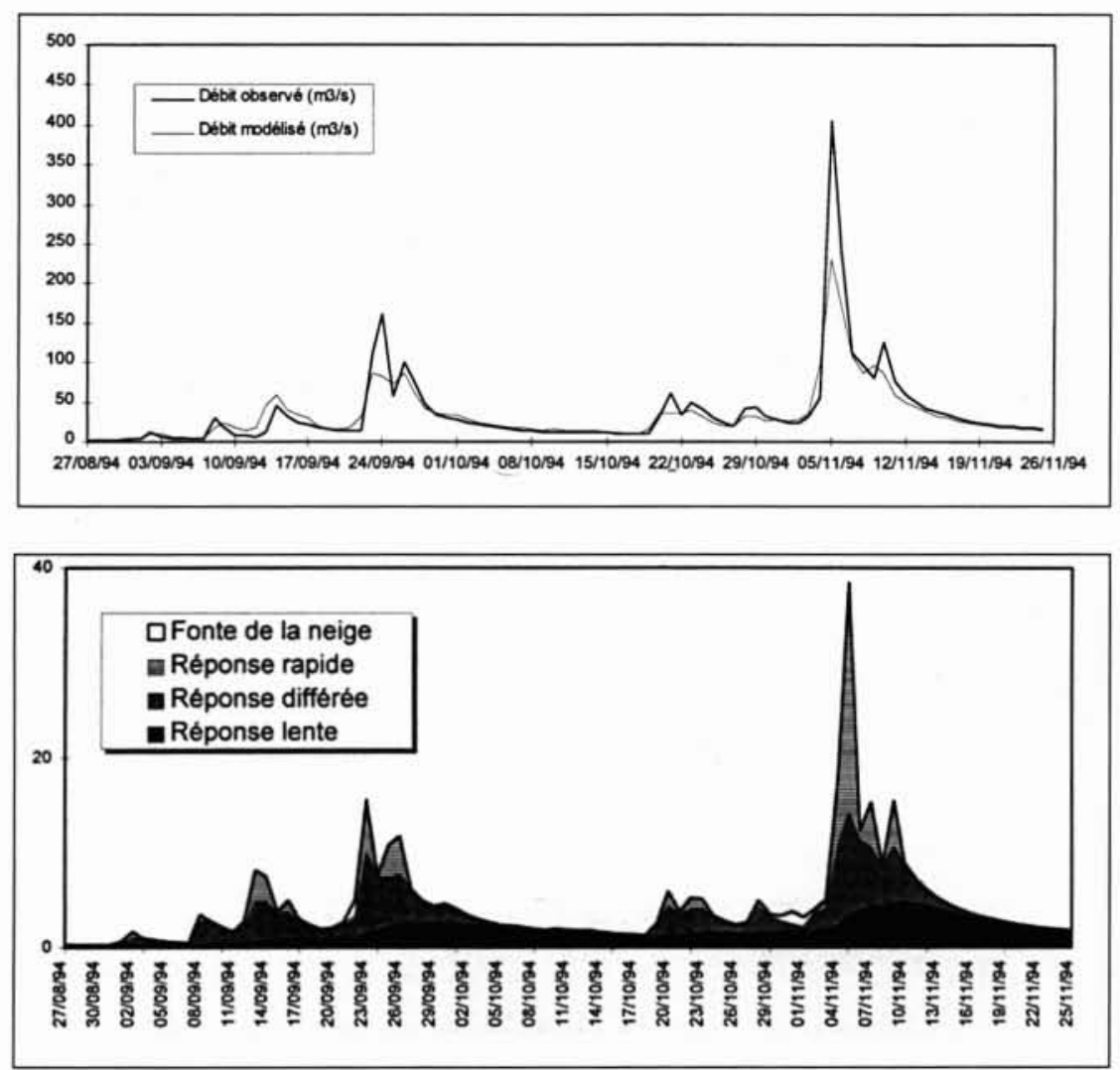

6. Simulation par MORDOR du débit du Verdon à Castillon. 
pluies, d'examiner la progression de l'écoulement d'un point de vue plus qualitatif. La production d'eau, telle que simulée dans MORDOR, a plusieurs origines conceptuelles et les contributions de chaque réservoir peuvent être mises en parallèle pour illustrer l'évolution du processus entre les crues normales et extrêmes. La figure 7 montre bien l'augmentation de la part relative de la réponse rapide dans l'écoulement pour les crues les plus fortes.

Bien que MORDOR ne soit pas un outil spécifiquement adapté aux crues, il nous a semblé intéressant de faire référence ici à un modèle à réservoirs, image conceptuelle populaire d'un bassin versant. Comment un réservoir peut-il simuler une réponse progressive du bassin de 5 à $80 \%$ comme celle illustrée par le tableau 2 ? Cela ne peut être le cas du réservoir conventionnel : un verre sous un robinet stocke $100 \%$ de ses apports jusqu'à ce qu'il soit plein, moment où le rendement marginal de sa production passe brutalement de 0 à $100 \%$. Mais un tel réservoir ne peut modéliser au mieux qu'une parcelle homogène très réduite dans un bassin rural. Sur l'ensemble du bassin, dans la réalité, certaines parcelles deviendront productives plus rapidement que d'autres, ce qui causera la progressivité de la réponse globale.

MORDOR rend compte de cette progression de la manière suivante : les 2 réservoirs qui gouvernent la dynamique des crues refusent les apports en eau au prorata de leur remplissage. Du coup, le rendement des pluies évolue progressivement entre 0 et $100 \%$, valeurs atteintes seulement dans les cas extrêmes où les réservoirs sont complètement secs ou, au contraire, saturés. Cette modélisation linéaire n'est bien sûr qu'une schématisation. Des représentations plus sophistiquées telles celle de TOPMODEL [10] font apparaître une non-linéarité importante. Mais, en première approximation, l'essentiel est d'éviter l'écueil d'un effet de seuil qui conduirait fatalement à un modèle trop sensible. On peut noter au passage que de tels réservoirs "à rendement progressif" donnent une réponse intégrée voisine de la fonction de production associée plus haut à la DPFT.

\section{V — SYNTHÈSE DES DIFFÉRENTES MODÉLISATIONS}

Nous avons examiné très brièvement le fonctionnement de 3 modèles qui, malgré leurs apparences et leurs objectifs variés, se rejoignent autour de grands principes.

\subsection{La production de l'écoulement rapide}

Sur le plan de la production de l'écoulement rapide à partir des pluies, les 3 modèles s'appuient sur l'idée d'un rendement marginal progressif tendant vers $100 \%$ pour les épisodes les plus importants. Cette idée générale peut sûrement être enrichie dans le détail mais elle permet, à elle seule, de rendre compte à $90 \%$ de l'évolution progressive entre le phénomène normal et l'événement extrême. Il n'a pas été besoin de seuil dans nos modèles pour assurer cette transition. A l'échelle du processus global, le concept de seuil semble beaucoup moins pertinent qu'à celle du mécanisme élémentaire local. Il faut bien comprendre que, sur des bassins ruraux assez vastes, même en montagne, même en climat méditerranéen, l'écoulement rapide des crues nor- males ne rassemble le plus souvent que quelques pour-cents de la pluie spatiale brute. Multiplions la pluie par 2, le rendement par 4 , et nous avons déjà un épisode extrême. Ce qui peut être brutal n'est pas tant l'évolution du processus hydrologique, lui-même relativement continu, que la survenue d'un événement 2 ou 3 fois plus important que ceux des 20 dernières années, au hasard d'un échantillonnage temporel capricieux, discontinu, au grand dam d'une espèce humaine manquant parfois de mémoire.

\subsection{La dynamique de l'écoulement}

Le second principe fédérateur de nos modèles concerne la dynamique temporelle de l'écoulement. Dans les 3 approches, nous avons implicitement considéré que l'on pouvait, en première approximation, se référer à une forme moyenne de fonction de transfert. Cette hypothèse simpliste a rendu bien des services même si, par exemple, l'on sait depuis toujours que le délai d'acheminement est raccourci quand l'épicentre de l'épisode pluvieux se rapproche de l'exutoire du bassin. De la même manière, l'on a des raisons physiques de soupçonner que la concentration par le réseau hydrographique est un peu plus rapide pour des débits élevés. Il n'en reste pas moins que l'étalement temporel de l'apport en eau à l'exutoire du bassin versant s'explique à $90 \%$ par ceux de la pluie spatiale et de la " fonction de transfert moyenne ". La distribution spatiale de la pluie et son intensité n'ont, comparativement, qu'un rôle mineur.

\subsection{Hydrologie opérationnelle et modélisation globale : et demain ?}

Le succès de la modélisation globale en hydrologie opérationnelle repose sur plusieurs atouts : simplicité des modèles et moindre gourmandise en données, complexité de la nature, imprécision dans les mesures ou dans leur représentativité spatiale ne valorisant pas les gains sur les détails, meilleure continuité à l'échelle du processus global qu'à celle du mécanisme local, permettant aux modèles de passer sans heurt de la normale à l'extrême...

Va-t-on pour autant en rester là ? Certainement pas. Une décennie de fiabilisation de la mise à disposition en temps réel de mesures de qualité a permis à l'hydrologie opérationnelle de progresser sans remettre en cause les bases méthodologiques de ses outils. Aujourd'hui, le radar météorologique dans certaines zones et l'automatisation des pluviographes ailleurs permettent à l'hydrologue de disposer en temps réel d'une meilleure connaissance de la distribution spatiale des dernières averses et l'incitent à utiliser cette information pour adapter en conséquence les fonctions de transfert et de production [11] de son bassin à l'épisode en cours. L'exploitation de telles informations doit pouvoir maintenant se généraliser.

Toutefois, il sera difficile, à court terme, d'aller beaucoup plus loin en hydrologie opérationnelle. Sans même envisager de coordonner, dans un même modèle, l'ensemble exhaustif des mécanismes physiques mis en jeu à une échelle fine pour reproduire le processus hydrologique global à l'échelle du bassin versant, l'idée, relativement simple, de découper un bassin en sous-éléments possédant leurs propres caractéristiques d'écoulement se heurte à un obstacle de taille. Sans une validation de la modélisation de chacun de ces éléments, l'apport d'une telle approche restera limité. On pourrait bien, 
en voulant appréhender le détail, ne faire que superposer les erreurs en perdant la maîtrise de l'ensemble. Seul un investissement important dans des mesures de terrain permettrait d'exploiter cette idée. Dans un contexte peu favorable à un tel investissement généralisé, on peut penser que la modélisation des crues en hydrologie opérationnelle subira à court terme une évolution, mais certainement pas une révolution dans ses bases méthodologiques.

\section{BIBLIOGRAPHIE}

[1] P. GUILLOT, D. DUBAND - 1967 - " La méthode du GRADEX pour le calcul de la probabilité des crues à partir des pluies " - IASH, publication $\mathrm{N}^{\circ} 84$

[2] CFGB - 1994 - " Les crues de projet des barrages : Méthode du gradex Design Flood Determination by the Gradex Method " - 18ème Congrès CIGB / ICOLD

[3] D. DUBAND, R. GARÇON - 1992 - " Extreme Precipitation Values and their Frequencies - Can we Rely on our estimates " - CIGB Grenade
[4] R. GARÇON - 1993 - " The Gradex Method : For a Statistically Robust Evaluation of Extreme Values Floods Based on Local Characteristics" . Stochastic and Statistical Methods in Hydrology and Environmental Engineering, Vol. 2, Kluwer Academic Publishers

[5] P. TOURASSE, 1. HUN - 1990 - " Evaluation des crues extrêmes du Verdon à Castillon et à Chaudane " - Note interne EDF

[6] P. GUILLOT, D. DUBAND - 1980 - * Une méthode de transfert pluiedébit par régression multiple " - IAHS $\mathrm{N}^{\circ} 129$

[7] D, DUBAND, I. NALBANTIS, C, OBLED, P. TOURASSE, J,Y, RODRIGUEZ - 1990 - " Unit Hydrograph revisited : the first differenced transfer function (FDTF) approach ". - IAHS N ${ }^{\circ} 190$

[8] J. PINTE, P. TOURASSE - 1981 - " Etude d'un modèle statistique de relation pluie-débit pour le Verdon à Castillon " - Note interne EDF

[9] R. GARÇON - 1996 - " Prévision opérationnelle des apports de la Durance à Serre-Ponçon à l'aide du modèle MORDOR " - La Houille Blanche $\mathrm{N}^{\circ} 5$

[10] K.J. BEVEN, R. LAMB, P. QUINN, R. ROMANOWICZ, J. FREER 1995 - " TOPMODEL, Computer Models of Watershed Hydrology " Water Ressources Publications

[11] R. DATIN - 1998 - " Outils opérationnels pour la prévision des crues rapides : traitement des incertitudes et intégration des prévisions météorologiques. Développements de TOPMODEL pour la prise en compte de la variabilité spatiale de la pluie. " - Thèse de I'INPG Grenoble. 